UDC 537.876

E. A. Bondarenko ${ }^{1}$, к.m.н., доиент

DOI: https://doi.org/10.20535/0203-3771402020249054

\title{
TWO BASIC SYSTEMS OF MAXWELL'S EQUATIONS IN A ROTATING FRAME: APPLICATION IN THEORY OF RING LASER GYRO
}

Ua У статті, в лінійному відносно кутової швидкості наближенні, розглянуто дві базові широко відомі системи рівнянь Максвела в рівномірно обертовій системі відліку. Першу систему рівнянь було вперше отримано в праці [L. I. Schiff, Proc. Natl. Acad. Sci. USA 25, 391 (1939)] на основі використання формалізму загальної теорії відносності, другу систему - в праці [W. M. Irvine, Physica 30, 1160 (1964)] на основі використання методу ортонормальних тетрад в цій теорії. У статті, в наближенні плоских хвиль, ці дві векторні системи рівнянь Максвела спрощено і записано в циліндричних координатах в компонентній формі 3 метою знайти закони поширення поперечних компонентів електромагнітних хвиль в круглому резонаторі лазерного гіроскопа у разі його обертання відносно осі чутливості. На основі цих двох спрощених систем рівнянь Максвела отримано широко відоме хвильове рівняння та його аналітичні розв'язки для вказаних поперечних компонентів хвиль. В результаті підстановки цих розв'язків в першу та в другу спрощені системи рівнянь Максвела встановлено, що вони задовольняють тільки другій із них. На цій підставі зроблено висновок про те, що друга система рівнянь Максвела більш підходить для застосування в теорії лазерного гіроскопа, ніж перша система.

Ru В статье, в линейном относительно угловой скорости приближении, рассмотрены две базовые широко известные системы уравнений Максвелла в равномерно вращающейся системе отсчета. Первая система уравнений была впервые получена в работе [L. I. Schiff, Proc. Natl. Acad. Sci. USA 25, 391 (1939)] на основе использования формализма общей теории относительности, вторая система - в работе [W. M. Irvine, Physica 30, 1160 (1964)] на основе использования метода ортонормальных тетрад в этой теории. В статье, в приближении плоских волн, эти две векторные системы уравнений Максвелла упрощены и записаны в цилиндрических координатах в компонентной форме с целью найти законы распространения поперечных компонентов электромагнитных волн в круглом резонаторе лазерного гироскопа в случае его вращения относительно оси чувствительности. На основе этих двух упрощенных систем уравнений Максвелла получено широко известное волновое уравнение и его аналитические решения для указанных поперечных компонентов волн. В результате подстановки этих решений в первую и во вторую упрощенные системы уравнений Максвелла установлено, что они удовлетворяют только второй из них. На этом основании сделан вывод о том, что вторая система уравнений Максвелла больше подходит для применения в теории лазерного гироскопа, чем первая система.

\footnotetext{
${ }^{1}$ КПІ ім. Ігоря Сікорського
} 


\section{Introduction}

The theory of ring laser gyro begins, as a rule, with a chapter devoted to an analysis of propagation of electromagnetic waves in its resonator. In order to perform such analysis, one needs to have the system of Maxwell's equations and corresponding system of wave equations for electromagnetic field vectors $\vec{E}$ and $\vec{B}$ in a frame of reference which uniformly rotates in an inertial space with angular velocity $\Omega(\Omega=|\vec{\Omega}|)$. Since the module $v$ of vector $\vec{v}=\vec{\Omega} \times \vec{r}$ of a tangential velocity of a rotating laser gyro is much smaller than the speed of light, it is sufficient for these systems to be linear in $v$ or, equivalently, in $\Omega$.

As an analysis of the literature shows, there are mainly two basic linear in $\Omega$ systems of Maxwell's equations for vectors $\vec{E}$ and $\vec{B}$ which are written in a uniformly rotating frame of reference. Both systems are based on the Galilean description of rotation: $t=t^{0}, \quad z=z^{0}, \quad x=x^{0} \cos \Omega t^{0}+y^{0} \sin \Omega t^{0}$, $y=-x^{0} \sin \Omega t^{0}+y^{0} \cos \Omega t^{0}$ (superscript ' 0 ' refers to the inertial frame).

In the case of vacuum, and in the absence of free charges and currents, the first system (first obtained in work [1] on the base of use of the formalism of the theory of general relativity) has the following form (we keep the terms only up to first order in $\Omega$ ):

$$
\begin{gathered}
\vec{\nabla} \times \vec{E}+\frac{\partial \vec{B}}{\partial t}=0, \\
\vec{\nabla} \cdot \vec{B}=0, \\
\vec{\nabla} \times\left(\vec{B}-\frac{1}{c^{2}} \vec{v} \times \vec{E}\right)-\frac{1}{c^{2}} \frac{\partial}{\partial t}(\vec{E}-\vec{v} \times \vec{B})=0, \\
\vec{\nabla} \cdot(\vec{E}-\vec{v} \times \vec{B})=0,
\end{gathered}
$$

and the second one (first derived in work [2] on the base of use of the method of orthonormal tetrad in this theory) is:

$$
\begin{gathered}
\vec{\nabla} \times \vec{E}+\frac{\partial}{\partial t}\left(\vec{B}+\frac{1}{c^{2}} \vec{v} \times \vec{E}\right)=0, \\
\vec{\nabla} \cdot\left(\vec{B}+\frac{1}{c^{2}} \vec{v} \times \vec{E}\right)=0, \\
\vec{\nabla} \times \vec{B}-\frac{1}{c^{2}} \frac{\partial}{\partial t}(\vec{E}-\vec{v} \times \vec{B})=0, \\
\vec{\nabla} \cdot(\vec{E}-\vec{v} \times \vec{B})=0
\end{gathered}
$$

Systems (1) and (2) contain only two electromagnetic field vectors $\vec{E}$ and $\vec{B}$. They are written here in the SI units. Systems (1) and (2), expressed in an- 
other units and containing, besides vectors $\vec{E}$ and $\vec{B}$, two more electromagnetic field vectors $\vec{D}$ and $\vec{H}$ (which must be excluded), may be found, for example, in subsequent works [3-24] and [25-27], respectively. Both systems and simple methods of their derivation are discussed in works [26, 28].

In systems (1) and (2), all the quantities are specified by the formulas

$$
\begin{gathered}
\vec{\nabla}=\hat{x}(\partial / \partial x)+\hat{y}(\partial / \partial y)+\hat{z}(\partial / \partial z), \\
\vec{E}=E_{x} \hat{x}+E_{y} \hat{y}+E_{z} \hat{z}, \quad \vec{B}=B_{x} \hat{x}+B_{y} \hat{y}+B_{z} \hat{z}, \\
\vec{\Omega}=\Omega_{x} \hat{x}+\Omega_{y} \hat{y}+\Omega_{z} \hat{z}, \quad \vec{r}=x \hat{x}+y \hat{y}+z \hat{z}, \\
\vec{v}=\vec{\Omega} \times \vec{r}=v_{x} \hat{x}+v_{y} \hat{y}+v_{z} \hat{z}, \\
v_{x}=\Omega_{y} z-\Omega_{z} y, \quad v_{y}=\Omega_{z} x-\Omega_{x} z, \quad v_{z}=\Omega_{x} y-\Omega_{y} x .
\end{gathered}
$$

Here $\hat{x}, \hat{y}$, and $\hat{z}$ are the unit vectors which form an orthogonal coordinate basis $\{\hat{x} \hat{y} \hat{z}\}$ of a rotating frame; $E_{x}, E_{y}$, and $E_{z}$ and $B_{x}, B_{y}$, and $B_{z}$ are the components of vectors $\vec{E}$ and $\vec{B}$ in this basis; $\vec{\Omega}$ is the vector of angular velocity with which basis $\{\hat{x} \hat{y} \hat{z}\}$ rotates in the inertial frame; $\Omega_{x}, \Omega_{y}$, and $\Omega_{z}$ are the components of vector $\vec{\Omega} ; \vec{r}$ is the radius-vector of the given observation point in basis $\{\hat{x} \hat{y} \hat{z}\} ; x, y$, and $z$ are the components of vector $\vec{r} ; \vec{v}$ is the vector of a linear tangential velocity of the observation point calculated in the inertial frame; $v_{x}, v_{y}$, and $v_{z}$ are the components of vector $\vec{v}$.

As we can see, the above two systems of Maxwell's equations (1) and (2) are qualitatively different: set (1) has asymmetrical structure with respect to $\Omega$ in the sense that rotation manifests itself only in the third and fourth equations but not in the first and second ones; set (2) has symmetrical structure with respect to $\Omega$ because rotation manifests itself in all four equations. As a consequence, the well-known substitutions $\vec{E} \rightarrow c \vec{B}$ and $\vec{B} \rightarrow-c^{-1} \vec{E} \quad$ (duality transformations) are possible only in system (2) but not in system (1).

One more additional detail: two corresponding systems of wave equations for vectors $\vec{E}$ and $\vec{B}$ obtained in work [29] on the base of sets of Maxwell's equations (1) and (2) are also qualitatively different. The first system [which was derived from set of Maxwell's equations (1)] has the form

$$
\begin{gathered}
\vec{\nabla}^{2} \vec{E}-\frac{1}{c^{2}} \frac{\partial^{2} \vec{E}}{\partial t^{2}}+\frac{2}{c^{2}} \frac{\partial}{\partial t}[(\vec{v} \cdot \vec{\nabla}) \vec{E}-\vec{\Omega} \times \vec{E}]-2 \vec{\nabla}(\vec{\Omega} \cdot \vec{B})=0, \\
\vec{\nabla}^{2} \vec{B}-\frac{1}{c^{2}} \frac{\partial^{2} \vec{B}}{\partial t^{2}}+\frac{2}{c^{2}} \frac{\partial}{\partial t}[(\vec{v} \cdot \vec{\nabla}) \vec{B}-\vec{\Omega} \times \vec{B}]=0,
\end{gathered}
$$

and the second one [which was obtained from set of Maxwell's equations (2)] is 


$$
\begin{aligned}
& \vec{\nabla}^{2} \vec{E}-\frac{1}{c^{2}} \frac{\partial^{2} \vec{E}}{\partial t^{2}}+\frac{2}{c^{2}} \frac{\partial}{\partial t}[(\vec{v} \cdot \vec{\nabla}) \vec{E}-\vec{\Omega} \times \vec{E}]-2 \vec{\nabla}(\vec{\Omega} \cdot \vec{B})=0, \\
& \vec{\nabla}^{2} \vec{B}-\frac{1}{c^{2}} \frac{\partial^{2} \vec{B}}{\partial t^{2}}+\frac{2}{c^{2}} \frac{\partial}{\partial t}[(\vec{v} \cdot \vec{\nabla}) \vec{B}-\vec{\Omega} \times \vec{B}]+\frac{2}{c^{2}} \vec{\nabla}(\vec{\Omega} \cdot \vec{E})=0 .
\end{aligned}
$$

From analysis of these expressions it follows that the structure of wave equations for vectors $\vec{E}$ and $\vec{B}$ in first system (3) is asymmetrical with respect to $\Omega$, while the structure of such equations in second system (4) is symmetrical. Again, as a consequence, the substitutions $\vec{E} \rightarrow c \vec{B}$ and $\vec{B} \rightarrow-c^{-1} \vec{E}$ are possible only in set (4) but not in set (3).

So from position of laser gyroscope, after taking these circumstances into account, we are standing before the problem of choice between systems of Maxwell's equations (1) and (2) [and, correspondingly, between sets of wave equations (3) and (4)]. In this situation we have to formulate the following principal question: which of the named two systems of Maxwell's equations, (1) or (2), is more suitable for application in the theory of ring laser gyro?

The concrete and reasonable answer to this question is not given in the literature ([1-28]) known to the author. So, in order to give the answer to it, - we must accept (as instrument for selection) a special physical criterion. In this paper, we propose the following one: analytical solutions of wave equations for components of vectors $\vec{E}$ and $\vec{B}$ must automatically satisfy the corresponding system of Maxwell's equations on the base of which these wave equations have been obtained.

So, the task of this paper is to give the answer to the named question on the base of use of the proposed criterion.

In order to solve the task, let us consider the ring laser gyro (see, for example, works $[20,26])$ with a planar polygonal empty $N$-mirror resonator of arbitrary shape with perimeter $L$ and area $A$ which provides generation of radiation linearly polarized in the sagittal plane $\left(\vec{E}=E_{z} \widehat{z}\right)$. In our calculations, after taking into account Stokes' theorem, it will be simpler to consider such gyro as if it has an equivalent hypothetical circular resonator of effective radius $\rho=2 \mathrm{~A} / L$. The device uniformly rotates about its sensitivity axis $\hat{z}$ (which is orthogonal to the meridional plane) with angular velocity $\Omega$, i.e., $\vec{\Omega}=\Omega \widehat{z}$. The gyro operates at central (of He-Ne active medium emission line) frequency $\omega_{0}$ (wavelength $\lambda_{0}=2 \pi c / \omega_{0}$, wavenumber $K_{0}=\omega_{0} / c=2 \pi / \lambda_{0}$ ). During the device operation on preselected at initial moment longitudinal mode with very large integer number $q$, the perimeter stabilization system of the gyro continuously provides (by adjusting parameter $\rho$ ) the fulfillment of resonance condition $2 \pi \rho=q \lambda_{0}$. 
On the base of consideration of such ring laser gyro, we must: (a) simplify and rewrite two systems of Maxwell's equations (1), (2) in scalar component form using the more convenient for our task cylindrical coordinates $\rho, \varphi$, and $z$; (b) obtain on the base of these two simplified systems of Maxwell's equations the corresponding wave equation for transversal components $E_{z}, B_{\rho}$ of propagating in laser resonator electromagnetic waves; (c) find from this wave equation the analytical solutions for quantities $E_{z}, B_{\rho}$; (d) substitute these solutions into the first and second simplified systems of Maxwell's equations and check how they satisfy these systems. After that, the final conclusion must be made giving the answer to the named question.

\section{Two simplified systems of Maxwell's equations for transversal components of vectors $\vec{E}$ and $\vec{B}$ in cylindrical coordinates}

For the above-mentioned ring laser gyro with a circular resonator of radius $\rho=2 A / L$, vectors $\vec{E}$ and $\vec{B}$ may be presented in cylindrical coordinates in the form $\vec{E}=E_{z} \hat{z}, \vec{B}=B_{\rho} \hat{\rho}$ Where $E_{z}$ and $B_{\rho}$ are the transversal components; $\bar{z}$ and $\hat{\rho}$ are the unit vectors. Since we consider the simplest special case of laser gyro rotation about its sensitivity axis $\bar{z}$ when $\vec{\Omega}=\Omega \hat{z}$, so $\vec{v}=v \hat{\varphi}$ where $v=\rho \Omega$, and $\hat{\varphi}$ is the unit vector.

Then, after introducing the more convenient for us longitudinal coordinate $s=\rho \varphi \quad(s=0, . ., 2 \pi \rho), \quad$ in the approximation of plane waves $\partial \vec{G} / \partial \rho=\partial \vec{G} / \partial z=0(\vec{G}=\vec{E}, \vec{B})$, and under simplifying condition $2 \pi \rho>>\lambda_{0}$ (see section 4 in [30]) of infinitely small curvature of a circular axis contour of laser gyro resonator (which always fulfils for modern devices with perimeter more than some centimeters), - we may present the operator $\vec{\nabla}$ in systems (1) and (2) in the form $\vec{\nabla}=\hat{\varphi}(\partial / \partial s)$. After that, the expressions for quantities $\vec{E}$, $\vec{B}, \vec{v}$, and $\vec{\nabla}$ must be substituted into these two systems, and their projections onto directions of unit vectors $\hat{\rho}$ and $\bar{z}$ must be taken.

As a result, the first and second simplified systems of Maxwell's equations (1) and (2) for wave transversal components $E_{z}, B_{\rho}$ will have in cylindrical coordinates correspondingly the following forms:

$$
\frac{\partial E_{z}}{\partial s}+\frac{\partial B_{\rho}}{\partial t}=0, \quad-\frac{\partial}{\partial s}\left(B_{\rho}-\frac{v}{c^{2}} E_{z}\right)-\frac{1}{c^{2}} \frac{\partial}{\partial t}\left(E_{z}+v B_{\rho}\right)=0,
$$

and

$$
\frac{\partial E_{z}}{\partial s}+\frac{\partial}{\partial t}\left(B_{\rho}+\frac{v}{c^{2}} E_{z}\right)=0
$$




$$
-\frac{\partial B_{\rho}}{\partial s}-\frac{1}{c^{2}} \frac{\partial}{\partial t}\left(E_{z}+v B_{\rho}\right)=0 \text {. }
$$

\section{Simplified wave equation for transversal components of vectors $\vec{E}$ and $\vec{B}$. Its analytical solutions}

A simplified wave equation for quantities $E_{z}, B_{\rho}$ may be obtained directly from approximate scalar systems of Maxwell's equations (5) and (6) by using the standard mathematical approach (via differentiation). As a result, this equation in first and second cases will have the following form:

$$
\frac{\partial^{2} G}{\partial s^{2}}-\frac{1}{c^{2}} \frac{\partial^{2} G}{\partial t^{2}}+\frac{2 v}{c^{2}} \frac{\partial^{2} G}{\partial s \partial t}=0 \quad\left(G=E_{z}, B_{\rho}\right) .
$$

The same expression (in order to be sure about its correctness) may be obtained directly from exact vectorial systems of wave equations (3) and (4) with the help of substitutions $\vec{E}=E_{z} \vec{z}, \quad \vec{B}=B_{\rho} \hat{\rho}, \quad \vec{\Omega}=\Omega \bar{z}, \quad \vec{v}=v \hat{\varphi}$, $\vec{\nabla}=\hat{\varphi}(\partial / \partial s)$.

Wave equation (7) must be solved with taking into account the boundary condition $G(s, t)=G(s+2 \pi \rho, t)$ where $2 \pi \rho=q \lambda_{0}$.

Equation (7) as well as its analytical solution for quantity $G=G(s, t)$ are well-known in the literature (see, for example, work [8] and formulas (80)-(85) therein). So, expressions for wave transversal components $E_{z}, B_{\rho}$ may be constructed as

$$
\begin{aligned}
& E_{z}(s, t)=E_{z 0} \cos \left(\omega_{1} t-K_{0} s\right)+E_{z 0} \cos \left(\omega_{2} t+K_{0} s\right), \\
& B_{\rho}(s, t)=B_{\rho 0} \cos \left(\omega_{1} t-K_{0} s\right)-B_{\rho 0} \cos \left(\omega_{2} t+K_{0} s\right),
\end{aligned}
$$

where $B_{\rho 0}=E_{z 0} / c, \omega_{1}=(1-\beta) \omega_{0}, \omega_{2}=(1+\beta) \omega_{0}, \quad \beta=v / c=\rho \Omega / c$,

$K_{0}=\omega_{0} / c=2 \pi / \lambda_{0}$.

Expressions (8) describe in mathematical form the lows of propagation of transversal electromagnetic waves in a uniformly rotating resonator of the laser gyro. According to (8), the difference $\omega_{\text {beat }}=\omega_{2}-\omega_{1}$ between frequencies of counterpropagating in its resonator waves may be calculated by the well-known (and experimentally confirmed with high accuracy) formula $\omega_{\text {beat }}=M_{g} \Omega$ where $M_{g}=8 \pi A /\left(\lambda_{0} L\right)$ is the so-called geometrical scale multiplier of the gyro. 
Result of substitution of expressions for transversal components of vectors $\vec{E}$ and $\vec{B}$ into the first and second simplified systems of Maxwell's equations

After substitution of expressions (8) for wave transversal components $E_{z}$, $B_{\rho}$ into simplified system of Maxwell's equations (5), we see that left-hand sides of these equations do not equal zero. In other words, analytical solutions (8) of wave equation (7) do not satisfy system (5) in the case $\Omega \neq 0$. This result is not acceptable for the theory of ring laser gyro because some doubts arise about correctness of relations (8).

At the same time, after substitution of expressions (8) into simplified system of Maxwell's equations (6), we see that left-hand sides of these equations do equal zero. That is, analytical solutions (8) of wave equation (7) do satisfy system (6) in the case $\Omega \neq 0$. Such result is acceptable for the theory of ring laser gyro because there are not any contradictions now between system of Maxwell's equations (6), wave equation (7) and its analytical solutions (8).

\section{Conclusion}

In the paper, using a linear in $\Omega$ approximation, two basic well-known systems of Maxwell's equations (1) and (2) for electromagnetic field vectors $\vec{E}$ and $\vec{B}$ in a uniformly rotating frame of reference are considered. The first system was first obtained in work [1] on the base of use of the formalism of the theory of general relativity, and the second one - in work [2] on the base of use of the method of orthonormal tetrad in this theory.

In the approximation of plane waves $\partial \vec{G} / \partial \rho=\partial \vec{G} / \partial z=0 \quad(\vec{G}=\vec{E}, \vec{B})$, and under the condition $2 \pi \rho>>\lambda_{0}$ (which always fulfills for modern ring laser gyros with perimeter more than some centimeters), these two vectorial systems of Maxwell's equations (1) and (2) are simplified and rewritten in cylindrical coordinates $\rho, \varphi$, and $z$ in scalar component form, correspondingly, (5) and (6), in order to find the lows of propagation of transversal components $E_{z}, B_{\rho}$ of electromagnetic waves in a circular resonator of laser gyro in the case of its rotation with angular velocity $\Omega$ about sensitivity axis $\widehat{z}$.

On the base of these two simplified systems of Maxwell's equations (5) and (6), the well-known wave equation (7) and its analytical solutions (8) for quantities $E_{z}, B_{\rho}$ are obtained. As a result of substitution of these solutions into the first and second simplified systems of Maxwell's equations (5) and (6), it is revealed that they satisfy only the second one.

Taking into account the special criterion for selection proposed in section 1 , that analytical solutions of wave equations for components of vectors $\vec{E}$ 
and $\vec{B}$ must automatically satisfy the corresponding system of Maxwell's equations on the base of which these wave equations have been obtained, - it may be considered that simplified system of Maxwell's equations (6) is preferable to simplified system (5).

But simplified set (6), in turn, has been derived from original system (2), while simplified set (5) has been obtained from original system (1). On this basis, the conclusion of the paper may be made that system of Maxwell's equations (2) is more suitable for application in the theory of ring laser gyro than system (1).

\section{References}

1. L. I. Schiff "A question in general relativity," Proc. Natl. Acad. Sci. USA 25, 391 (1939).

2. W. M. Irvine, "Electrodynamics in a rotating system of reference," Physica 30, 1160 (1964).

3. J. Ise and J. L. Uretsky, "Vacuum electrodynamics on a merry-go-round," Am. J. Phys. 26, 431(1958).

4. D. L. Webster, "Schiff's charges and currents in rotating matter," Am. J. Phys. 31, 590 (1963).

5. C. V. Heer, "Resonant frequencies of an electromagnetic cavity in an accelerated system of reference," Phys. Rev. 134, A799 (1964).

6. A. Yildiz and C. H. Tang, "Electromagnetic cavity resonances in accelerated systems," Phys. Rev. 146, 947 (1966).

7. A. M. Khromykh, "Ring generator in a rotating reference system," Soviet Physics JETP 23, 185 (1966).

8. E. J. Post, "Sagnac effect," Rev. Mod. Phys. 39, 475 (1967).

9. J. L. Anderson and J. W. Ryon, "Electromagnetic radiation in accelerated systems," Phys. Rev. 181, 1765 (1969).

10. G. E. Modesitt, "Maxwell's equations in a rotating reference frame," Am. J. Phys. 38, 1487 (1970).

11. A. M. Volkov and V. A. Kiselev, "Proper frequencies of a rotating ring resonator," Soviet Physics JETP 30, 733 (1970).

12. L. D. Landau and E. M. Lifshitz. The Classical Theory of Fields. Pergamon Press, New York.1971.

13. T. Shiozawa, "Phenomenological and electron-theoretical study of the electrodynamics of rotating systems," Proc. IEEE 61, 1694 (1973).

14. S. G. Zeiger, Yu. L. Klimontovich, P. S. Landa, E. G. Lariontsev, and E. E. Fradkin. Wave and Fluctuation Processes in Lasers. Nauka, Moscow. 1974. 
15. H. A. Atwater, "The electromagnetic field in rotating coordinate frames," Proc. IEEE 63, 316 (1975).

16. G. Lianis and D. Whicker, "Electromagnetic phenomena in rotating media," Arch. Rat. Mech. and Anal. 57, 325 (1975).

17. A. A. Korostelev and V. F. Fateev, "Electrodynamics of moving media in noninertial reference systems as applied to the processes in a ring resonator," Optika i Spektroskopiia 45, 132 (1978).

18. I. V. Shpak and A. V. Solomin, "Effect of the refractive index of the medium on the splitting of the eigenfrequencies of a rotating ring cavity," Optics and Spectroscopy 46, 72 (1979).

19. O. Gron, "Application of Schiff's rotating-frame electrodynamics," Int. J. Theor. Phys. 23, 441 (1984).

20. W. W. Chow, J. Gea-Banacloche, L. M. Pedrotti, V. E. Sanders, W. Schleich, and M. O. Scully, "The ring laser gyro," Rev. Mod. Phys. 57, 61 (1985).

21. A. Georgiou, "The electromagnetic field in rotating coordinates," Proc. IEEE 76, 1051(1988).

22. G. C. Scorgie, "Electromagnetism in non-inertial coordinates," J. Phys. A: Math. Gen. 23, 5169 (1990).

23. T. Numai, "Beat frequencies in a ring laser gyro with its refractive index over unity," J. Appl. Phys. 89, 1537 (2001).

24. B. Z. Steinberg, A. Shamir, and A. Boag, "Two-dimensional Green's function theory for the electrodynamics of a rotating medium," Phys. Rev. E 74, 016608 (2006).

25. T. C. Mo, "Theory of electrodynamics in media in noninertial frames and applications," J. Math. Phys. 11, 2589 (1970).

26. L. N. Menegozzi and W. E. Lamb, Jr., "Theory of a ring laser," Phys. Rev. A 8, 2103 (1973).

27. N. D. Milovskii, "Equations of electrodynamics in a rotating solid dielectric," Optics and Spectroscopy 123, 642 (2017).

28. K. T. McDonald, "Electrodynamics of rotating systems" (Princeton University, 2008);

http://physics.princeton.edu/ mcdonald/examples/rotatingEM.pdf.

29. E. A. Bondarenko, "Two systems of Maxwell's equations and two corresponding systems of wave equations for electromagnetic field vectors $\vec{E}$ and $\vec{B}$ in a rotating frame of reference: a linear approximation," Ukr. J. Phys. 62, 99 (2017).

30. E. A. Bondarenko, "Two first-order systems of Maxwell's equations for electromagnetic field vectors $\vec{E}$ and $\vec{B}$ in a rotating frame of reference: 


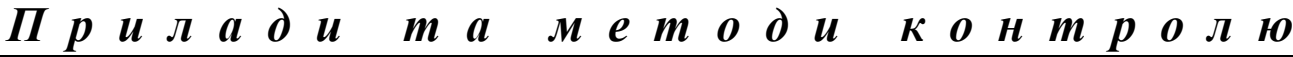

which one is more suitable for description of rotation?" (ResearchGate, 2018); https://www.researchgate.net/publication/323186607. 